\title{
Running before we can walk?
}

\author{
Two years ago, a new \\ proteomic test was \\ heralded as the future \\ of cancer diagnostics.
} But since then, doubts about its effectiveness have begun to grow. Erika Check reports.

\section{$\mathrm{S}$} eldom does a single piece of research prompt the US Congress to pass a resolution urging continued funding to drive a new diagnostic test towards the clinic. But that's what happened in 2002, when The Lancet published a paper ${ }^{1}$ claiming a breakthrough in the diagnosis of ovarian cancer.

The paper described the use of mass spectrometry to analyse the pattern of proteins present in samples of blood serum. On the basis of these patterns, the test detected all the patients with ovarian cancers in a set of 50 samples, and falsely identified just three healthy patients as suffering from the disease from a total of 66 control samples.

Most encouragingly, the technique seemed to work well on patients with earlystage cancer - offering the prospect of earlier diagnosis, which improves the chances of successful treatment. The best current blood test, which relies on the detection of a single protein called CA125, misses at least half of patients in the earliest stages of the disease, and gives a high rate of false positives.

The researchers, led by Lance Liotta and Emanuel Petricoin, who co-direct a proteomics programme run by the National Cancer Institute and the Food and Drug Administration, based in Bethesda, Maryland, won immediate acclaim. In addition to the congressional resolution urging further funding for their research, the consumer magazine Health named the test one of the top ten medical advances of the year.

Commercial rights to develop the test were quickly licensed from the US government to a company called Correlogic Systems, also based in Bethesda, whose scientists collaborated with Liotta and Petricoin on the

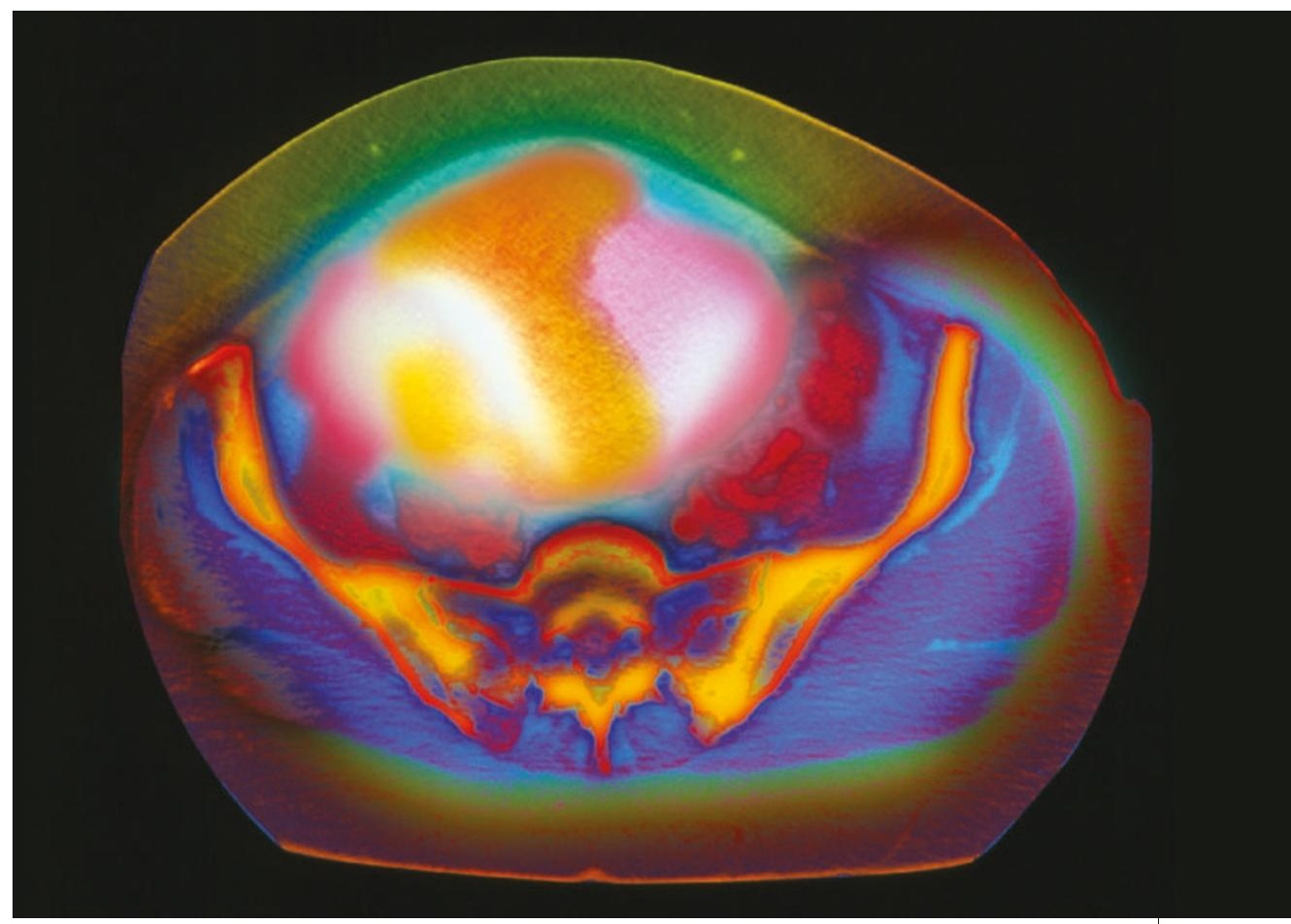

On target: can proteins in the blood reveal ovarian tumours (pink/yellow) before they reach this stage?

Lancet paper. In November 2002, Correlogic granted licences to two larger firms, Quest Diagnostics and the Laboratory Corporation of America, which are now hoping to market the test under the brand name OvaCheck.

But those plans could be thrown off track by reanalyses of Liotta and Petricoin's data by independent groups, which have raised serious doubts about OvaCheck's reliability.

These questions prompted the Society of Gynecologic Oncologists to review all of the published work about OvaCheck. On 7 February, the society declared that "more research is needed to validate the test's effectiveness before offering it to the public".

\section{Early warning}

Critics warn that the episode illustrates the dangers of moving rapidly to the clinic with immature technologies such as those of proteomics. They say that scientists and regulators need to develop standards to

"Whether or not
OvaCheck works, we
will learn from this
experience what rules
of evidence we might
apply in the future to
find useful results
more efficiently."
ensure that such tests really work before they hit the market, because early detection is not without risks. Women who get false positive results may undergo unnecessary surgery, and those who get false negatives may forgo further screening. "Early detection is not a benign undertaking," says Martin McIntosh, who runs a cancer-detection effort at the Fred Hutchinson Cancer Research Center in Seattle, Washington.

The first criticisms of OvaCheck hit the public domain in June 2003, when two biostatisticians at the University of Maryland in Baltimore, James Sorace and Min Zhan, published a paper in the online journal $B M C$
Bioinformatics ${ }^{2}$. They had reanalysed a data set that Liotta and Petricoin's team posted online in August 2002. Sorace and Zhan similarly found numerous differences in the protein patterns that discriminated between the cancer patients and the healthy controls. The trouble, according to Sorace and Zahn, was that these looked more like experimental artefacts than real biological differences.

The proteomics test relies on using gravity and electric fields to separate the proteins in a given sample. Each protein is then given a number that represents the ratio of its charge and mass - called its $\mathrm{m} / z$ value. The test identifies patterns in these numbers to give a diagnosis. Sorace and Zahn were concerned because the most marked differences between the cancer patients and unaffected controls occurred for proteins with $\mathrm{m} / \mathrm{z}$ values of less than 500. Many spectrometry experts consider $\mathrm{m} / \mathrm{z}$ values below 2,000 to be suspect, because they tend to include values generated by experimental artefacts or measurement error. To Sorace and Zahn, this suggested that the data for cancer patients and controls had been collected under such different conditions that it was impossible to reliably identify true biological differences.

Further questions emerged when biostatisticians led by Keith Baggerly of the MD Anderson Cancer Center in Houston, Texas, reanalysed the data in the Lancet paper, plus two further data sets posted online. "Based on these data, we really can't tell if it's possible to use proteomics to separate normals from cancers," Baggerly says. 
